\title{
BRAIN MAPPING
}

\section{Dysfunctional Default Mode Network during high speed discrimination in the elderly. Neuropsychological and driving simulator correlations.}

\begin{tabular}{|r|l|}
\hline Journal: & Human Brain Mapping \\
\hline Manuscript ID & Draft \\
\hline Wiley - Manuscript type: & Research Article \\
\hline Date Submitted by the Author: & n/a \\
\hline Complete List of Authors: & $\begin{array}{l}\text { Eudave, Luis; Centro de Investigacion Medica Aplicada, Neuroimaging } \\
\text { Martinez, Martin; Center for Applied Medical Research, University of } \\
\text { Navarra School of Medicine, Department of the Neurological Sciences } \\
\text { Luis, Elkin; Centro de Investigacion Medica Aplicada, Neuroimaging; } \\
\text { University of Navarra, School of Education and Psychology } \\
\text { Pastor, Maria; Center for Applied Medical Research, University of Navarra } \\
\text { School of Medicine, Department of the Neurological Sciences }\end{array}$ \\
\hline Keywords: & $\begin{array}{l}\text { high speed discrimination, default mode network, elderly, aging, driving } \\
\text { simulator, fMRI, functional connectivity }\end{array}$ \\
\hline \multicolumn{2}{|c|}{} \\
\hline
\end{tabular}




\title{
Research Article:
}

\section{Dysfunctional Default Mode Network during high speed \\ discrimination in the elderly. Neuropsychological and driving simulator correlations.}

\author{
Luis Eudave, ${ }^{1}$ Martín Martínez, ${ }^{1}$ Elkin O. Luis,,${ }^{1,2}$ María A. Pastor ${ }^{1}$ \\ 1. Neuroimaging Laboratory, Division of Neurosciences, Centre for Applied Medical \\ Research (CIMA), University of Navarra, 31008, Pamplona, Spain. \\ 2. School of Education and Psychology, University of Navarra, Pamplona, Spain.
}

Short title: Aging correlates in high speed discrimination

Keywords: high speed discrimination, default mode network, elderly, aging, driving simulator, fMRI, functional connectivity

Correspondence should be addressed to: Maria A. Pastor, Neuroimaging Laboratory, Division of Neurosciences, Centre for Applied Medical Research (CIMA), University of Navarra, 31008, Pamplona, Spain (e-mail: mapastor@unav.es). 
Eudave et al.

\section{Abstract}

Numerous daily tasks, including car driving, require fine visuospatial tuning. One such visuospatial ability, speed discrimination, declines with aging but its neural underpinnings remain unknown. In this study, we use fMRI to explore the effect of aging during a high speed discrimination task, along with a complete neuropsychological assessment and a simulated driving evaluation in order to examine how they interact with each other. Beyond confirming that high speed discrimination performance is dimished in the elderly, we found that this deficit might be partly due to a lack of modulation in the activity and connectivity of the default mode network (DMN) in this age group, as well as an over-recruitment of frontal, basal ganglia and cerebellar regions, possibly as a compensatory mecanism. This neural pattern could also be translated to our participants' cognitive and driving simulator performance, such that in young adults, a proper DMN modulation correlated with better neuropsychological scores and a driving profile, an effect that seems to be lost in the elderly. These findings contribute to highlight the role of the default mode network on visuospatial tasks, how it is age-related and its impact on cognitive functioning and driving performance in a simulator. 


\section{Introduction}

\subsection{On speed discrimination and its age-related changes}

Speed discrimination can be defined as the ability to detect and recognize the differences in speed of moving objects, a more complex task than speed detection and processing (McKee, 1981). Despite speed being the rate of change in spatial position, and therefore containing two measurable variables -time and space- it has been proposed that speed can be perceived as a separate attribute, subject to discrimination (Lappin, Bell, Harm, \& Kottas, 1975).

Speed discrimination tasks are generally used to determine a subject's speed discrimination threshold. To this end, moving light bars (Orban, de Wolf, \& Maes, 1984), random-dot patterns (de Bruyn \& Orban, 1988; Orban et al., 1998) or moving grating patterns (Snowden \& Kavanagh, 2006; Sunaert, Van Hecke, Marchal, \& Orban, 2000) are presented as a two-alternative forced-choice procedure in order to detect the threshold were the subject is no longer able to differentiate between two stimuli with different speeds, which can be as low as $5 \%$ (McKee, 1981).

Age-related differences in speed discrimination have shown mixed results. One of the first studies comparing speed discrimination in age groups, Brown and Bowman (Brown and Bowman, 1987) did not find differences between young and older adults, with stimuli moving from 3 to $8.8 \%$ s. In contrast, Norman et al. (2003) found that in the elderly, the ability to discriminate between two moving stimuli is deteriorated by up to a $71 \%$, with females presenting worse scores compared to males. This effect was further confirmed by Raghuram et al. (2005) who found higher thresholds in older women in speed discrimination when shorter stimuli $(500 \mathrm{~ms})$ were presented. 
Eudave et al.

Most studies have employed relatively slow stimuli, with only a few using higher speeds (>64\%) (de Bruyn \& Orban, 1988; Orban et al., 1984). At these speeds, the detectable Weber fraction seems to increase by up to $15 \%$ in young adults, representing the ascending arm of an U-shaped speed function, where stimuli become harder to discriminate, a finding that leads us to think that the difference in the elderly could be even wider with a high speed discrimination (HSD) task. This is important to take into account, since naturalistic stimuli, like those found in everyday activities such as car driving, move at higher speeds and can comprise real life risks.

\subsection{Neural correlates of speed discrimination}

There has been little research exploring the neural correlates of speed discrimination. In one experiment, using fluorodeoxiglucose positron emission tomography (FDG-PET) in using a "speed identification" minus "detection" paradigm, found differential activation in the right cuneus, bilateral lingual gyri, along with the left anterior cingulate and bilateral frontal operculum (Orban et al., 1998). In a similar experiment with BOLD-fMRI and using a moving random texture pattern, Sunaert et al. (2000), replicated these findings, reporting activation in the cuneus bilaterally, left and right lingual gyri and the supplementary motor area (SMA). In another experiment where attention to speed was compared to coherence of motion, differential activity in the V3 and V3a cortices, posterior cingular cortex (PCC), precuneus, inferior parietal lobule (IPL) and medial prefrontal cortex was found (Kau et al., 2013).

These neuroimaging studies have all examined speed discrimination in a young healthy population, and as of yet no work has been done on the elderly. Since this population is susceptible to changes in speed discrimination, a change in functional 
Eudave et al. activity and connectivity can be expected. The Default Mode Network (DMN), comprised of different frontal, parietal and temporal structures is particularly affected in the elderly, showing differences in activation and connectivity across several cognitive tasks, which are usually represented as a lack of deactivation while executing a task (Grady et al., 2010; Park, Polk, Hebrank, \& Jenkins, 2010) or as a decline in functional connectivity (Andrews-Hanna et al., 2007; Dørum et al., 2016; Persson, Pudas, Nilsson, \& Nyberg, 2014) or efficiency, changes that can even be found in middle-aged adults (Siman-Tov et al., 2017).

\subsection{Driving simulators and the elderly}

Differences in simulated driving performance between young and older adults have been widely documented, where impairments in the elderly are usually observed (Bélanger, Gagnon, \& Stinchcombe, 2015; Campagne, Pebayle, \& Muzet, 2004; Lee, Cameron, \& Lee, 2003; Stinchcombe, Gagnon, Zhang, Montembeault, \& Bedard, 2011; Urwyler et al., 2015). Other studies have focused on the relationship between simulated driving performance in the elderly and cognitive processes such as visual attention (Cuenen et al., 2015; Hoffman, McDowd, Atchley, \& Dubinsky, 2005), divided attention, mental workload (Cantin, Lavallière, Simoneau, \& Teasdale, 2009), processing speed, visuospatial performance, executive functioning (Shanmugaratnam, Kass, \& Arruda, 2010) suggesting either deficits in these abilities or the existence of a possible underlying compensatory effect (Andrews \& Westerman, 2012). An extension on how speed estimation or speed discrimination affect real or simulated driving hasn't been fully explored, despite it being an ability affected by aging. In an attempt to link driving and speed estimation, participants seated in the passenger seat were asked to estimate on-going car changes in velocity (Scialfa, Guzy, Leibowitz, Garvey, \& Tyrrell, 1991), finding that older adults 
Eudave et al. showed less sensitivity to changes in velocity, a finding that might be related to traffic accidents (Hills, 1980).

Cognitive dysfunction has been also related to car crashes and accident rates (Bélanger, Gagnon, \& Yamin, 2010) or dangerous driving in a simulator (Roenker, Cissell, Ball, Wadley, \& Edwards, 2003). This diminished or worse simulated driving performance does not necessarily imply impaired real driving, especially since driving simulators usually use oversimplified scenarios and also due to the fact that volunteers know the experience is not real and therefore do not carry the risks that real driving does. One approach to solve this problem has been the validation of simulated driving by comparing it with an on-drive evaluation, which has demonstrated some resemblance between modalities (Lee et al., 2003) even when observing age-related driving fluctuations (Aksan et al., 2016). However, it has been reported that driving simulator training has a positive impact on cognitive performance (Casutt, Theill, Martin, Keller, \& Jäncke, 2014) and also on on-road driving abilities (Roenker et al., 2003), supporting the use of driving simulators as a rehabilitation tool.

Hence, here we aim to explore the age-related changes on brain activity and functional connectivity during a HSD task, and how brain activity is related with different cognitive domains and with driving performance, as measured in a driving simulator. Our hypothesis is then threefold: (1) that the elderly group will perform worse in the HSD task, which then will be reflected in a change in activity and functional connectivity when compared to a younger control group; (2) that our nondemented elderly group will show signs of cognitive decline associated with performance in HSD; and that (3) differences in driving performance between groups will be related to performance in the HSD task. 
Eudave et al.

\section{Materials and Methods}

\subsection{Participants}

A total of 49 volunteers were recruited and classified in two groups: 24 healthy young subjects (YS) and 25 healthy older subjects (OS). Seven participants were excluded from the study (2 YS, 5 OS) due to incomplete examinations (2), distress during the MRI scan (2), possible cognitive impairment (2) and an abnormal MRI scan (1). The final number of evaluated subjects was 22 for YS (11 males, $30.3 \pm 4.3$ years old [ descriptive results will continue to be presented in the mean \pm standard deviation format]) and 20 for OS (14 males, $67.4 \pm 5.2$ years old).

Inclusion criteria for the YS group included subjects aged from 20 to 40 years old and a valid driving license; for the OS group, an age of more than 60 years was required, along with a valid driving license. Exclusion criteria included those associated with MRI use (metallic implants, claustrophobia, etc.), the presence of cognitive dysfunction or another neurological disease, current central nervous system modulating treatment and abnormal findings on the subjects' structural MRI scan.

All participants were right-handed (mean dexterity score $47.4 \pm 3.3$ out of 50 ) as determined by the 10 item Edinburgh handedness inventory (Oldfield, 1971). Each item was scored from 1 to 5 . The total score was calculated by adding the individual item scores. A total high score indicates right-handedness and a total low score, lefthandedness.

Volunteer recruitment was mainly done at the University of Navarra, through its studentship and alumni. All but 2 YS were research-naive, and had never participated in any study before. The experimental protocol was approved by the 
Eudave et al.

University of Navarra Research Ethics Committee. Subjects signed a written informed consent before participating in the study.

\subsection{Neuropsychological evaluation}

Additionally, we were also interested in running a complementary neuropsychological examination to test for cognitive fitness of our populations, as well as to use these results to correlate them with neuroimaging data. This examination included the followings tests: Mini-Mental Status Exam to rule out cognitive impairment; the Geriatric Depression Scale (GDS) for depressive symptoms; Trail Making Test A and B (TMT-A, TMT-B) to evaluate visual attention and divided attention; Stroop Effect test for sustained attention and inhibition; the Digit Span, both forward and backward, to test for short-term and working memory; Ray Auditory Verbal Learning Test which examines verbal memory span, long-term memory, verbal learning, and verbal recognition; the Digit Symbol Coding (from the WAIS-IV) test for processing speed index; the Weschler Memory Scale test for short and long-term visual reproduction; the Phonological and Semantic Fluency tests; the Block Design test that measures perceptual organization; and the "Zoo Map" or Behavioral Assessment of the Dysexecutive Syndrome (BADS) test that examines planning and problem solving abilities.

In order to facilitate analysis, these tests were classiffied in six cognitive domains: (1) Perceptual organization (Block Design test), (2) Attention (TMT-a, TMT-b, Stroop Effect test), (3) Verbal Cognition (Ray Auditory Verbal Learning Test and forward Digit Span test), (4) Visual Cognition (Block Design test and Weschler Memory Scale test), (5) Language (Phonological and Semantic Fluency tests) and (6) Executive (TMT-b, backwards Digit Span test, Stroop Effect test, Digit Symbol Coding test and "Zoo Map" test). In order to facilitate visualization of differences between groups, t- 
Eudave et al.

tests results were normalized to z-scores. Also, a composite score was made for each domain by averaging individuals' scores in the corresponging tests in order to build a variable that could be evaluated during further neuroimaging analyses.

\subsection{Driving simulator setup and evaluation}

The driving simulator set-up used in this study (Signos, Prometeo Innovations C) consisted of a PC, a 40-inch TV, the Logitech G25 Driving Wheel, Pedals and Stick and a racing seat. The screen was positioned $1 \mathrm{~m}$ in front on the driver while seated and displayed a simulated first person view from the inside of a Toyota Yaris driver's seat. The driving session consisted of a $40 \sim$ minute evaluation where participants had to follow a set of pre-defined traffic indications by a virtual driving instructor through a 3-stage circuit (Supplementary Figure 1) while driving as they would in a real car. The first stage took place in an urban environment that included traffic lights, different speed limits, pedestrians crossing the street, slow traffic and roundabout crossing that lasted for approximately $2.4 \mathrm{~km}$. The second stage was done on a highway where participants had to drive for $11.6 \mathrm{~km}$ (round trip) with a 120 $\mathrm{km} / \mathrm{h}$ speed limit. In the third stage participants had to drive through a two-lane mountain road (9.6 km, roundtrip) with traffic and different speed limits. Participants were continually monitored for simulator sickness symptoms during the whole evaluation.

A total of 26 telemetric parameters were registered during each driving evaluation, which were related to how fast the participant was driving (total session time, \% of time moving, \% of time above speed limit, mean speed at 40, 50, 80, 100 and 120 $\mathrm{km} / \mathrm{h}$ ), pedal management (time with pedal $>75 \%$, $\%$ of time with pedal $>75 \%$, time braking, $\%$ of time braking, time with brake pedal $>75 \%$, $\%$ of time with brake pedal $>75 \%$, number of $>5$ second brakes, number of $>10$ second brakes), steering 
Eudave et al.

(number of $60-90^{\circ}, 90-180^{\circ}, 180-270^{\circ}, 270-360^{\circ}$ steers in 0.5 seconds) and traffic violations (two wheel sidewalk invasion, collision with other cars or objects, yellow or red traffic light skips and runovers). After each driving session a log file including these parameters was created, from which data was then extracted and analysed.

\section{4 fMRI experimental setup and design}

Subjects laid supine inside the scanner. Images were projected onto a screen and reflected by a mirror system attached to the head coil into the subjects' field of vision from which stimuli speeds were calculated. Motor finger responses were collected from a 4-key response pad (Current Designs, Inc.), placed on the subjects' abdomen. Stimuli design, presentation and data collection were done using PsychoPy2 v1.82.01 (Peirce, 2008) and Matlab (R2017b, The Mathworks Inc., Natick, MA, USA).

The objective of this experiment design was for the subject to discriminate between two visual stimuli presented sequentially with different high velocities. Thus, we asked individuals to answer which of them was faster. The experimental task consisted of a pseudo block event-related design which included rest, control and task conditions (Figure 1). Each cycle (14 in total) started with an 8 second Rest period where subjects had to maintain visual fixation at the central cross. Task blocks consisted of four trials in which a white $\left(2.5^{\circ}\right.$ radius $)$ circle crossed the screen from left to right in the superior visual hemifield followed by another circle crossing from left to right in the inferior visual hemifield. One of the following eleven speeds was pseudorandomly assigned to each stimuli: $106 \%$ s, $113 \%$ s, $120 \%$ s, $127 \%$, $134 \%, 141 \%$ s, $148 \%$ s, $155 \%$, 162\%, 169\%, 176\%. Speed difference (SDF) was defined as the difference in speed between two stimuli, giving rise to either a positive (first stimulus faster than the second by $7 \%$ s, $21 \%$ s, $28 \%$, $42 \%$ s, $56 \%$ or $70 \%$ s) or 
negative (second stimulus faster than the first by $-14 \%,-21 \% \mathrm{~s},-28 \% \mathrm{~s},-36 \% \mathrm{~s},-42 \% \mathrm{~s}$, or $-56 \%$ ) value. The subject was given $2,000 \mathrm{~ms}$ to indicate which of both stimuli was the fastest, by pressing the first button on the response pad if they thought it was the first stimulus or by pressing the second button if it was the second stimulus. Subsequently, another 8-second Rest period was displayed, followed by the Control block which consisted of 4 equal trials of a single white square $\left(2.5^{\circ}\right.$ per side) crossing the screen from left to right at a speed of $106 \%$ after which the subject had to press any button in response to the stimulus detection. Responses were recorded for further offline performance analysis. The duration of Task and Control blocks was of 16 seconds each, making up for a total of 48 seconds per cycle. The total run duration was 11 minutes and 12 seconds.

\section{Figure_1}

\subsection{Speed discrimination performance data}

In this study, two behavioral variables were assessed: Accuracy (AC), which was defined as the average number of correct responses per trial; and Response Time (RT), defined as the time it took the participants to give an answer per trial. Mean AC and RT values were compared between groups and were also analysed by SDF in order to examine how the difference in speed between stimuli impacted performance.

\subsection{Behavioral statistical analysis}

Scores and variables from neuropsychological evaluation and experimental tasks were compared between groups through Mann-Whitney $U$ tests, due to non-normal data distribution of many variables, along with its effect size calculated by rankbiserial correlation. Post-hoc resulting p-values in the neuropsychological tests (20 in 
Eudave et al. total) and driving simulator telemetric variables (26 in total) were corrected for multiple comparisons using the Bonferroni method. Also, two 2-way ANOVAs (with factors Group and SDF) were conducted to test for the effect of AC and RT when at different speeds.

To understand the relationship between the series of variables delivered by our driving simulator, we performed an exploratory factor analysis test which included the 26 telemetric variables in order to find the main factors that could better explain data variance. Specifically we ran a parallel analysis, with orthogonal rotation (varimax) and a loading threshold of 0.6. All behavioral statistical analyzes were conducted on the JASP software (JASP Team, 2017).

\section{7 fMRI data acquisition and analysis}

BOLD fMRI studies were performed on a 3.0 Tesla MR scanner (Siemens TRIO, Germany) using a 16-channel head coil. A total of 210 whole-brain functional volumes using a $\mathrm{T}^{*}$-weighted gradient echo-planar imaging (EPI) sequence (repetition time/echo time $[\mathrm{TR} / \mathrm{TE}]=3000 / 30 \mathrm{~ms}$, field of view $[\mathrm{FOV}]=192 \times 192$ $\mathrm{mm}^{2}$, flip-angle $=30^{\circ}, 48$ slices, resolution $=3 \times 3 \times 3 \mathrm{~mm}^{3}$ ) were acquired during each session in an interleaved fashion. A total of three "dummy" volumes were discarded due to scanner stabilization.

The anatomical image was obtained using a whole-brain T1-weighted MPRAGE sequence $(\mathrm{TR} / \mathrm{TE}=1620 / 3 \mathrm{~ms}$, inversion time $(\mathrm{TI})=950 \mathrm{~ms}, \mathrm{FOV}=250 \times 187 \times 160$ $\mathrm{mm}^{3}$, flip-angle $=15^{\circ}, 160$ slices, resolution $=1 \times 1 \times 1 \mathrm{~mm}^{3}$ ). No fat suppression was employed.

Mass univariate data analysis was done using SPM12 (r6225, Wellcome Department of Imaging Neuroscience, UCL, London). To detect severe motion or artifacts we 
Eudave et al.

completed preliminary visual checks. Conventional preprocessing pipeline steps were followed. First, EPI images were motion-corrected and realigned to the first volume of the series and co-registered to the anatomical image. Slice time correction followed, taking slice 25 (first) as the reference slice. Functional and anatomical images were then normalized to the coordinates of the MNI (Montreal Neurological Institute) template, version ICBM-152. A three-dimensional Gaussian smoothing kernel of $8 \mathrm{~mm}$ full width at half maximum (FWHM) was applied to the EPI images.

Statistical analysis was performed following the General Linear Model (GLM) and modelled with the canonical double gamma hemodynamic response function (HRF). At the first-level analysis, two Conditions (Task, Control) were modeled for all subjects in both Groups (YS, OS). Six motion regressors were included to the design matrix to control for any head movements generated during image acquisition. All subjects' Task>Control contrasts (contrast of interest) were obtained and exported for further analysis.

Second-level analyses comparing contrasts of interest between groups were done using two-sample t-tests, where differences in brain activity between Groups (YS>OS, OS>YS) and mutual coactivation (Global conjunction, positive and negative YSกOS) were obtained using a primary threshold of $p<0.001$ and corrected for multiple comparisons using Family Wise Error (FWE, $\mathrm{p}<0.05)$ method at the cluster level. Anatomical cerebral activations were defined at the peak activation maxima and percent signal change differences were extracted using the Anatomy toolbox (v2.0) for all significant clusters.

\subsection{ROI definition and functional connectivity analysis}

Using Marsbar, two different sets of regions of interest (ROI) were created: one for the conjunct activation (positive YSกOS) contrast and the second for the conjunct 
Eudave et al.

deactivation (negative YSกOS) contrast, where common clusters between both groups were taken into account to test for differences in connectivity. Seeds were defined as $5 \mathrm{~mm}$-spheres centered at the peak MNI coordinates for each resulting cluster.

Task>Control data was analysed using the CONN 16.b Functional Connectivity Toolbox. Conventional analysis steps were followed: BOLD data smoothing $(8 \mathrm{~mm})$, despiking and bandpass filtering for low frequency signals $(0.008-0.1 \mathrm{~Hz})$. White matter and cerebrospinal fluid and movement parameters BOLD signals were regressed out. Also, Task-related coactivations and effects were removed from the signal in order to avoid correlations.

We conducted a series of Seed-to-Voxel analyses for each ROI, corrected by False Discovery Rate (FDR $\mathrm{p}<0.05)$ at the cluster level. This method allowed us to explore the positive and negative functional connectivity between the selected ROI and all voxels in our volumes.

\subsection{Neuroimaging vs behavioral data analysis}

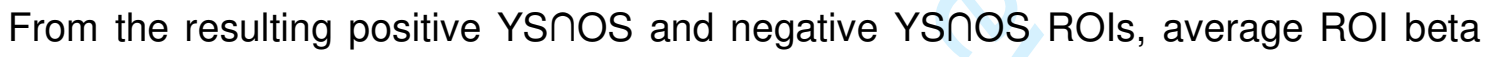
values were extracted for each subject using the Marsbar toolbox in order to assess how brain activity was related to behavioral performance in HSD and also to the obtained neuropsychological and driving simulator factors.

Likewise, from the results of the functional connectivity analyses, average connectivity values within supra-threshold clusters were extracted for each seed and subject, and compared against the aforementioned behavioral measures and factors using linear regression models. 


\section{Results}

\subsection{Demographic results}

Demographic results are detailed in Table I. Significant differences were found for age and driving experience but not for gender, nor the MMSE and GDS tests.

Table_I

\subsection{Neuropsychological results}

Neuropsychological results are shown in Figure 2 and detailed in Table I. Perceptual organization was not significant between groups. However, differences in the Attention domain were significant between groups in all three visual, sustained and divided attention tests. In the Verbal Cognition domain, significant differences were found only for long-term memory but not for memory span, short-term memory, verbal learning nor verbal recognition. On Visual Cognition, significant differences between groups (that did not surpass Bonferroni correction) were found in memory span and long-term memory, but not for short-term memory. No differences were found for either phonological or semantic fluency in the Language domain. In the Executive domain, significant differences were found for mental flexibility, problem solving (did not surpass Bonferroni correction), inhibition and processing speed but not for planning nor working memory.

Figure_2

\subsection{Driving simulator results}

Results on the driving simulator performance are detailed in Table II. One YS and five OS participants were excluded from this analysis due to simulator sickness that 
Eudave et al.

impeded evaluation completion. Also, four YS and three OS subjects reported mild simulator sickness symptoms (i.e., eye strain, dizziness).

The exploratory factor analysis indicated that only 20 variables (with no crossloadings) surpassed the loading threshold, which was intentionally set high due to the relative small number of variables (Supplementary Table I). The Bartlett's Test of Sphericity was significant $\left(x^{2}(205)=633.35, p<0.001\right)$ demonstrating the adequacy of our data. A total of five uncorrelated factors were identified that explained $71 \%$ of the variability in data (Supplementary Figure 2). The relationship between variables within each factor allowed for adequate labeling: driving Speed, which included variables related to the time it took the subject to finish the driving session, the speed while driving and yellow light skips; Dexterity, which included sudden steering at any angle; Collision Risk for gas pedal pressing parameters and collisions with other cars; and Hard Braking and Braking for hard brake pressing and time braking parameters respectively. From these factors and their variables, we created composite scores in order to compare these results between groups and to use them as covariates in our functional neuroimaging analysis. These scores were based on the sum of the mean value of every variable in each factor, weighted by the loading value.

When compared by factors, only Speed was significantly different between groups, indicating that YS were faster during the driving session. Within this factor, YS had a lower total session time, less yellow traffic light skips, higher \% of time above speed limit, and mean speed at $40 \mathrm{~km} / \mathrm{h}, 50 \mathrm{~km} / \mathrm{h}, 80 \mathrm{~km} / \mathrm{h}$ and $120 \mathrm{~km} / \mathrm{h}$ zones. Besides these, only the number of two wheel sidewalk invasions variable was also significantly different between groups. From all results, only the total session time 
and mean speed at $120 \mathrm{~km} / \mathrm{h}$ zone variables surpassed the Bonferroni correction threshold.

\section{Table_II}

\subsection{HSD performance results}

Results from AC and RT mean values compared by group and by SDF are summed up in Figure 3. AC was significantly lower in the OS group. When errors in $A C$ were analysed by the speed difference between stimuli, main effects of Group and SDF and interactions were statistically significant. Further post-hoc analyses showed, as expected, that more errors were committed in both groups when the difference between speeds was minimal. Interestingly, OS committed most errors when the second stimulus presented was faster (Figure 3c), being significantly higher than those on the YS group.

Figure_3

When evaluating the effect of sex in AC (Supplementary Figure 3), significant main effects of Group and Sex were found but no interactions. Post-hoc comparisons showed that only in our OS group women made more errors that men (Wos: $14.5 \pm$ 3.73, Mos: $9.43 \pm 3.29, t=4.46, p=0.016)$.

Alternatively, no differences between groups were found in mean RT. When further analysed, only the SDF factor was significant $(F[11,440]=5.44, p<0.001)$, with no main effect of Group nor interactions.

\subsection{Neuroimaging results}

\subsubsection{Global activity}


Eudave et al. Maps of differential and conjunction activation analysis are summarized in Tables III and IV respectively. The OS>YS contrast revealed a widespread hyperactivation pattern (Figure 4), peaking in the SMA, right insula, precuneus, left dorsolateral prefrontal cortex (DLPFC), fusiform gyri, left temporal pole, left thalamus, left angular gyrus and cerebellum (see Supplementary Figure 4 for changes in percent signal change).

\section{Table_III}

The positive YSกOS contrast showed common activity in the DMPFC, right DLPFC, bilateral intraparietal sulcus and bilateral insulae. The negative YSกOS contrast showed activity in areas that configure the DMN, including the medial prefrontal cortex, precuneus, PPC and left middle temporal gyrus along the left angular gyrus.

\section{Figure_4}

\subsubsection{Functional connectivity results}

Results of functional connectivity analyses are shown in Figure 5 and detailed in Table IV. In the positive YSกOS analysis, the YS group showed an increased connectivity between the right precentral gyrus with nearby right frontal areas, mostly SMA. OS was found to have higher connectivity than YS in two ROIs: the right insula with the contralateral putamen and insula; the right SMA with right posterior parietal (angular gyrus, supramarginal gyrus), lateral occipital cortex, temporal (inferior, middle and superior temporal gyrus) and also with the left thalamus.

\section{Figure_5}

In the negative YSกOS, the YS group showed a generalized higher connectivity than OS between DMN regions: the superior medial gyrus with the precuneus and PCC; the middle cingulate cortex (MCC) connected with frontal medial cortex, PCC, 
Eudave et al.

precuneus, pericingulate and left middle temporal cortex (MT); the PCC with the precuneus, frontal medial cortex and left MT; the precuneus with the frontal medial cortex, left MT, lateral occipital cortex, both angular gyri and both temporal poles; the MT connected with the precuneus, PCC and right and left cuneal cortices; and finally the left angular gyrus was connected to the anterior cingulate cortex (ACC), precuneus, left paracingulate and lingual gyri. Also, YS had a stronger connectivity between the left mid orbital gyrus the left frontal cortex and pole. On the inverse contrast, OS only revealed a higher connectivity than YS between the left MT and left frontal pole.

\section{Table_IV}

\subsection{Neuroimaging vs behavioral data analysis}

\subsubsection{HSD task performance}

When comparing $\mathrm{AC}$ (measured in errors) against every $\mathrm{ROI}$ average beta values (Table V), in YS we found a significant negative correlation (less errors, increased activation) in positive conjunction areas (left inferior parietal lobule, right inferior parietal lobule left insula, right insula, right inferior frontal gyrus, right precentral gyrus and right SMA). Conversely, we found a significant correlation for OS but in negative conjunction DMN areas (medial frontal cortex, MCC, PCC, precuneus).

Contrasting against connectivity values (Table VI), YS showed a significant positive correlation (more errors, more connectivity) between the precentral gyrus and the medial frontal gyrus and a negative correlation (less errors, more connectivity) between the left middle temporal gyrus and the PCC. The OS group revealed only a negative correlation between the right SMA and the left thalamus.

\subsubsection{Neuropsychological correlations}


Eudave et al.

In YS (Table V), better cognitive outcomes in neuropsychological domains correlated significantly with an increase in activity in positive conjunction ROls, for the Attention (right supramarginal gyrus) and Executive (right inferior frontal gyrus) domains respectively, along with a decrease in activation in negative conjunction ROls for the the Verbal (left precuneus, right precuneus) and Perception (left middle orbital gyrus and left middle temporal gyrus) domains. On the contrary, a worse score in OS in the Executive domain correlated significantly with an increase in activation in the right inferior parietal lobule.

\section{Table_V \\ Table_VI}

When compared to functional connectivity values (Table VI), neuropsychological correlations were more heterogeneous: YS showed a significant correlation between better scores and higher DMN connectivity within the Attention (precuneusprecuneus, left middle temporal gyrus-precuneus), and Language (left middle temporal gyrus-precuneus, PCC-medial frontal gyrus) domains; correlations in OS between better scores and higher connectivity were found only in the Language domain (right SMA-right angular gyrus, left SMA-right middle temporal gyrus).

\subsubsection{Driving simulator correlations}

In YS, a lower Speed score (faster driving) correlated with increased brain activation (Table V) in the left angular gyrus; a higher Dexterity with increased activity in the left middle orbital cortex; a higher Collision Risk score with left insula activiation; an increased Braking score with activity in the right inferior frontal gyrus, left SMA, right SMA and right supramarginal gyrus; and higher Hard Braking scores with higher superior parietal lobule and lower scores with left middle orbital gyrus, and left 
Eudave et al. middle temporal gyrus activity. The OS group also revealed a significant correlation between an increased activation and higher Braking scores in the left inferior parietal lobule and higher Collision Risk in the superior medial frontal cortex.

When compared to higher connectivity average values (Table VI), YS only correlated with a higher Dexterity score and connectivity between the superior medial frontal cortex and precuneus; in the OS group, high connectivity values correlated with lower Speed scores between the right SMA and right angular gyrus; and with higher Collision Risk values and connectivity between right SMA and left thalamus. 
Eudave et al.

\section{Discussion}

In this study we demonstrate the presence of a deficit associated to high speed discrimination in the elderly when compared to a young adult control group, in spite of a wide frontoparietal and cerebellar hyperactivation pattern, in conjunction with reduced DMN deactivation and connectivity within its areas. Moreover, we found that neurocognitive fitness and several driving parameters correlated with adequate DMN functioning in young adults, an effect that appears to be lost with aging. Finally, an age-dependant relationship between brain activity and HSD performance and collision risk was established.

\subsection{The Default Mode Network is dysfunctional in the elderly while executing a high speed discrimination task}

Our speed discrimination paradigm revealed a common between-group set of activated/deactivated areas, as well as differential activation when examined separately by group. Common group activations were found in the bilateral insulae and right DLPFC, areas usually found to in task-positive experiments (Cabeza \& Nyberg, 2000); also the bilateral intraparietal sulcus (IPS) and the supplementary eye fields (SEF), structures that conform the dorsal attention network (Corbetta \& Shulman, 2002). These areas are involved in goal directed (top-down) control, by supporting visuo-spatial attention and decision making through retention in shortterm spatial memory which may participate in correct discrimination between moving stimuli. Deactivation in both groups was detected in DMN regions (ventral precuneus, medial frontal cortex) an effect usually present in task-positive paradigms (M E Raichle et al., 2001; Marcus E. Raichle, 2015). 
Eudave et al. Hyperactivation of the elderly group when compared with young adults is widespread, and included the SMA, ACC, left inferior frontal gyrus, dorsal precuneus, bilateral basal ganglia, fusiform gyrus and the cerebellum. These changes were characterized by a rise in activation in the aforementioned areas along with a diminished deactivation of the dorsal precuneus. All of these results are in consonance with the compensation hypothesis for the aging brain (P.A. ReuterLorenz \& Campbell, 2008; Patricia A. Reuter-Lorenz \& Park, 2014) which proposes that frontal and parietal overactivation, along with DMN reduced deactivation, may act as a compensation measure, needed to reach youth-like levels of performance until it reaches a maximum and starts to decline. Usually, these changes in activation are in close relationship with changes in behavioral performance assessments, which were also corroborated in this study since higher accuracy correlated with DMN activity and connectivity in young adults.

Besides examining similarities and differences in group activation, we also aimed to compare the functional connectivity patterns emerging from areas common to both age groups. From the positive conjunction analysis, the young group showed a stronger connectivity than the elderly between the precentral gyrus and SMA; in the inverse contrast, the elderly had stronger connections than the young group between the SMA and the PPC and right temporal cortex; and the right insula with the contralateral insula and putamen. From negative conjunction analyses, we found a loss of connectivity between several DMN areas in the the elderly (precuneus, PPC, posterior and anterior cingulate) when compared to the young group. This DMN connectivity dysfunction has been found in other cognitive tasks such as spatial jugdement tasks (Park et al., 2010), object tracking (Dørum et al., 2016), semantic classification (Andrews-Hanna et al., 2007), working (Grady et al., 2010; Sambataro et al., 2010) and episodic memory (Persson et al., 2014). It appears that the DMN 
Eudave et al.

loses efficiency as a network, since it is not able to deactivate appropriately and connections between components are weakened when compared to a young DMN.

\subsection{High speed discrimination accuracy is impaired in the elderly}

In our high speed discrimination task, subjects had to compare two stimuli moving at high speeds and choose the fastest one. As expected, more errors were committed when the difference between stimuli was minimal, with a pronounced contrast between younger and older participants, hence confirming previous findings (Norman et al., 2003; Raghuram et al., 2005) and demonstrating its effect when discriminating at higher speeds. Moreover, the elderly group made most of its errors when the speed difference was negative, meaning that they incorrectly chose the first stimulus as the fastest when it was the second, especially when the speed difference between stimuli was reduced. When the opposite was true (second faster than the first) they performed similarly to the young group. This finding could be related to the fact that at the beginning of each trial, the elderly were not as attentive as the young, and therefore a fast first stimulus might have come as "unexpected", leading to confusion to decide whether that stimulus was indeed the fastest. This attentional deficit is corroborated by the older group scores in the visual, sustained and divided attention tests.

Also, we found sex-related differences in our elderly group, where accuracy in females was lower. This effect has been found in previous speed discrimination studies but with slower stimuli (Norman et al., 2003; Raghuram et al., 2005), as well as in other direction discrimination studies (Atchley \& Kramer, 1998; Gilmore, Wenk, Naylor, \& Stuve, 1992); these differences have generally been attributed to men having better visuo-spatial abilities, especially when dealing with moving stimuli, although differences might be minimal and depend on the task at hand (Ardila, 
Eudave et al.

Rosselli, Matute, \& Inozemtseva, 2011). In line with this, our results must be taken cautiously due to the unequal number of males and females (14 and 6, respectively) in the elderly group.

Mean RT was similar between groups which could explained by the fact that subjects were explicitly requested to try and achieve maximum accuracy, and not to answer as fast as they could. Also, no interactions were found in the two-way ANOVA suggesting that RT does not change with aging independently of the speed differences between stimuli. Despite this, the plot shows a qualitative difference between groups in some speed difference ranges $(-58 \% / \mathrm{s},-42 \% / \mathrm{s})$ suggesting that a significant interaction might exist (at least some ranges) if we increase the sample size.

\subsection{Task performance and neurocognitive status correlates with a proper functioning DMN}

Correlations between brain activity and task performance showed that in the young subjects group, higher Accuracy in the high speed discrimination test directly correlates with increased activation in frontoparietal areas, such as the precentral gyrus, inferior frontal gyrus and the bilateral inferior parietal lobuli and the insulae. This relationship between task performance and frontoparietal activation in the young has also been found in several working memory tasks (Kaup, Drummond, \& Eyler, 2014; Leung, Oh, Ferri, \& Yi, 2007; Nagel et al., 2009). In contrast, unlike the trend seen in young adults, our elderly group presented an inverse correlation between Accuracy and deactivation of the DMN (precuneus, MCC, PCC, frontal medial cortex), suggesting that instead of normally deactivating the DMN, the elderly tend to recruit (deactivate less) those areas, possibly as compensation, in order to properly perform this task. Contrary to our results, studies examining working 
Eudave et al.

memory and spatial judgement tasks in aging found that better performance correlated with greater deactivation (Kennedy, Boylan, Rieck, Foster, \& Rodrigue, 2017; Prakash, Heo, Voss, Patterson, \& Kramer, 2012), proposing that adequate DMN modulation may be a sign of maintained cognitive ability in aging (Rieck, Rodrigue, Boylan, \& Kennedy, 2017). The disparity in our results could be explained by 1 ) the fact that these studies took task difficulty (and creating a "difficulty" contrast of interest) into account when assessing performance and 2) that since performance in the young group also did not correlate with the DMN in our task, it might be an indication that the elderly, instead of efficiently employing frontoparietal areas, have to rely on DMN activation to preserve good performance.

When looking into the correlation between connectivity values and performance, we found that in young adults, better Accuracy correlated with higher connectivity between DMN areas (left MT and PCC), and lower connectivity between the right precentral gyrus and frontal gyrus. In exchange, better Accuracy in the elderly correlated with higher connectivity values between the SMA and left thalamus. This finding suggests that in the young, besides having a higher overall within DMN connectivity, this increment also relates to task performance, whereas in the elderly, better Accuracy is achived through the intervention of fronto-thalamic resources, both facts compatible with the elderly compensatory scaffolding theory (P.A. ReuterLorenz \& Campbell, 2008).

In regard to the neuropsychological scores, better scores correlated significantly with an adequate brain activity response in young adults, such that better scores in Attention and Executive correlated with the amount of activity in the supramarginal gyrus and inferior frontal cortex respectively, and scores in Verbal and Perception correlated with the appropriate deactivation of DMN structures (precuneus and 
Eudave et al.

middle temporal gyrus). Also, better scores in the Attention and Language composites correlated with higher connectivity within the DMN areas. This demonstrates how better cognitive performance is associated with the usage of positively activated areas, but maybe more importantly with the DMN. These findings were not present in the elderly; activation of the inferior parietal lobule, normally a positively activated area, was correlated only with worse Executive scores. Also, only higher connectivity values in connections between the SMA and angular gyrus and MT correlated with better Language scores in the elderly. This is further evidence of how age related decreases in DMN activity and connectivity may affect cognitive functioning and how frontal areas get involved in order to give an "extra hand" when DMN modulation is diminished or absent.

\subsection{Older subjects drive slower and the give-and-take between acceleration and car collisions that is modulated differently with aging}

Our driving simulator results revealed faster driving in the young adult group. In an attempt to explore and classify driving performance, an Exploratory Factor Analysis was done, which resulted in five main factors that could be grouped in five drivingrelated dimensions: Speed, Hard Braking and Braking, all of these directly related to speed and time; Dexterity, which measured the degree of sudden driving wheel turns; and Collision Risk, which included high acceleration and car collisions. Such measures might represent good indicators of driving performance and profiling, as well as a tool to evaluate responses to challenging driving events (Bélanger et al., 2010). Differences in groups were only found in the Speed factor, where the young were found to drive faster overall. These results confirm previous findings on simulated driving where older adults drive at slower (Cantin et al., 2009; Lee, Drake, \& Cameron, 2002; Shanmugaratnam et al., 2010) and more variable speeds 
Eudave et al.

(Urwyler et al., 2015), which tend to be even slower than in on-road assessments (Lee et al., 2003).

Driving simulator performance values and their correlation with brain BOLD activity was heterogeneous: in young adults, higher DMN deactivation correlated with higher Speed, Dexterity and Hard Braking measures, while higher activity in frontoparietal areas (SMA, posterior parietal cortex) was associated with higher Braking values. Interestingly, high activity in the left insula correlated with higher Collision Risk values, a region that also correlated with higher accuracy in our high speed discrimination task. Connectivity wise, only a positive correlation between Dexterity and the connection between medial frontal cortex and the precuneus was found.

Higher activation in the elderly correlated with higher Braking values in the inferior parietal cortex and with higher Collision Risk in the medial frontal cortex; the latter comparison is relevant since a correlation between this area and better accuracy in the speed discrimination task was also found, suggesting that a decreased deactivation in a normally deactivated DMN area helps the elderly to better discriminate, while also being related to higher acceleration when driving, which in turn might have led to collisions with other cars in our simulator. The apparent compromise between better high speed discrimination/high acceleration and the potential risk of car collisions, might then be distinctively modulated with aging, by changing from the employment of attentional resources from the insula in the young, to compensating through the use of the medial frontal cortex in the elderly.

A similar scenario was seen when we compared connectivity values and driving performance in the elderly: we found a correlation between lower Speed and higher connectivity between the SMA and angular gyrus, and between the SMA and left thalamus with higher Collision Risk values, a connection that was also associated 
Eudave et al. with better high speed discrimination accuracy. This enhanced fronto-thalamic connection serves as another link between high speed discrimination and the Collision Risk factor in the elderly, where improved discrimination and higher acceleration may come at the risk of car collisions. To summarize it can be extracted that in the young, different driving factors can interact with either positive or negatively modulated areas, while in the elderly these interactions are not as well defined, although an association was found between higher Collision Risk values and higher DMN activation and fronto-thalamic connectivity, changes also related to better high speed discrimination.

\subsection{Limitations}

As with other driving simulator studies, our simulator might have introduced bias since it does not fully represent the act and experience of driving, therefore altering how our participants approach and execute the driving task. Also, several of our subjects presented simulator sickness, a feature intrinsic to virtual simulators due to perceptual (visual, vestibular, proprioceptive) mismatch. 
Eudave et al.

\section{Conclusions}

In summary, our study delivers evidence on how high speed discrimination is impaired in an otherwise healthy older adult population and how this alteration is associated with a widespread hyperactivation pattern that included an increase in activity in frontal areas, the basal ganglia and the cerebellum, along with reduced deactivation in DMN areas and a loss of connectivity within DMN areas. Better performance in the young group correlated with higher frontoparietal activity and higher within DMN connectivity; in the elderly higher Accuracy correlated with a loss of DMN deactivation and higher SMA-thalamic connectivity. In young adults, better cognitive function correlated higher frontoparietal activity (Attention, Executive), higher DMN deactivation (Verbal, Perception) and connectivity (Attention, Language); in the elderly this modulation is lost. Finally, our driving simulator task showed that in the young, depending on the driving factor, either frontal or DMN activation and connectivity play a more active role; meanwhile in the elderly, a correlation was found between acceleration and collision risk and DMN activation/fronto-thalamic connectivity, neural patterns that were also correlated with better high speed discrimination. In conclusion, the age-related DMN dysfunction found in this study intervenes in high speed discrimination performance and healthy aging, conditions that may also be reflected in simulated driving behaviors. 


\begin{abstract}
Acknowledgments
This work was funded by the Fundación Caja Navarra grant (Ref: FBCN 15/70757) for biomedical research. LE was financially supported by a grant from the Univesity of Navarra Friends Association. The authors declare no conflict of interest.
\end{abstract}


Eudave et al.

\section{References}

Aksan, N., Hacker, S., Sager, L., Dawson, J., Anderson, S., \& Rizzo, M. (2016). Correspondence between Simulator and On-Road Drive Performance: Implications for Assessment of Driving Safety. Geriatrics, 1(1), 8. https://doi.org/10.3390/geriatrics1010008

Andrews-Hanna, J. R., Snyder, A. Z., Vincent, J. L., Lustig, C., Head, D., Raichle, M. E., \& Buckner, R. L. (2007). Disruption of Large-Scale Brain Systems in Advanced Aging. Neuron, 56(5), 924-935. https://doi.org/10.1016/j.neuron.2007.10.038

Andrews, E. C., \& Westerman, S. J. (2012). Age differences in simulated driving performance: Compensatory processes. Accident Analysis and Prevention, 45, 660-668. https://doi.org/10.1016/j.aap.2011.09.047

Ardila, A., Rosselli, M., Matute, E., \& Inozemtseva, O. (2011). Gender differences in cognitive development. Developmental Psychology, 47(4), 984-990. https://doi.org/10.1037/a0023819

Atchley, P., \& Kramer, a F. (1998). Spatial cuing in a stereoscopic display: attention remains "depth-aware" with age. The Journals of Gerontology. Series B, Psychological Sciences and Social Sciences, 53(5), P318-P323.

Bélanger, A., Gagnon, S., \& Stinchcombe, A. (2015). Crash avoidance in response to challenging driving events: The roles of age, serialization, and driving simulator platform. Accident Analysis and Prevention, 82, 199-212. https://doi.org/10.1016/j.aap.2015.04.030

Bélanger, A., Gagnon, S., \& Yamin, S. (2010). Capturing the serial nature of older 
drivers' responses towards challenging events: A simulator study. Accident Analysis and Prevention, 42(3), 809-817.

https://doi.org/10.1016/j.aap.2009.07.010

Brown, B., \& Bowman, K. J. (1987). Sensitivity to changes in size and velocity in young and elderly observers. Perception, 16(1), 41-47.

Cabeza, R., \& Nyberg, L. (2000). Imaging Cognition II: An Empirical Review of 275 PET and fMRI Studies. Journal of Cognitive Neuroscience, 12(1), 1-47. https://doi.org/10.1162/08989290051137585

Campagne, A., Pebayle, T., \& Muzet, A. (2004). Correlation between driving errors and vigilance level: Influence of the driver's age. Physiology and Behavior, 80(4), 515-524. https://doi.org/10.1016/j.physbeh.2003.10.004

Cantin, V., Lavallière, M., Simoneau, M., \& Teasdale, N. (2009). Mental workload when driving in a simulator: Effects of age and driving complexity. Accident Analysis and Prevention, 41(4), 763-771. https://doi.org/10.1016/j.aap.2009.03.019

Casutt, G., Theill, N., Martin, M., Keller, M., \& Jäncke, L. (2014). The drive-wise project: Driving simulator training increases real driving performance in healthy older drivers. Frontiers in Aging Neuroscience, 6(MAY), 1-14. https://doi.org/10.3389/fnagi.2014.00085

Corbetta, M., \& Shulman, G. L. (2002). Control of Goal-Directed and Stimulus-Driven Attention in the Brain. Nature Reviews Neuroscience, 3(3), 215-229. https://doi.org/10.1038/nrn755

Cuenen, A., Jongen, E. M. M., Brijs, T., Brijs, K., Lutin, M., Van Vlierden, K., \& Wets, G. (2015). Does attention capacity moderate the effect of driver distraction in 
Eudave et al.

older drivers? Accident Analysis and Prevention, 77, 12-20.

https://doi.org/10.1016/j.aap.2015.01.011

de Bruyn, B., \& Orban, G. A. (1988). Human velocity and direction discrimination measured with random dot patterns. Vision Research, 28(12), 1323-1335. https://doi.org/10.1016/0042-6989(88)90064-8

Dørum, E. S., Alnæs, D., Kaufmann, T., Richard, G., Lund, M. J., Tønnesen, S., ... Westlye, L. T. (2016). Age-related differences in brain network activation and co-activation during multiple object tracking. Brain and Behavior, (February), 115. https://doi.org/10.1002/brb3.533

Gilmore, G. C., Wenk, H. E., Naylor, L. a, \& Stuve, T. a. (1992). Motion perception and aging. Psychology and Aging, 7(4), 654-660. https://doi.org/10.1037/08827974.7.4.654

Grady, C. L., Protzner, A. B., Kovacevic, N., Strother, S. C., Afshin-Pour, B., Wojtowicz, M., ... McIntosh, A. R. (2010). A multivariate analysis of age-related differences in default mode and task-positive networks across multiple cognitive domains. Cerebral Cortex, 20(6), 1432-1447.

https://doi.org/10.1093/cercor/bhp207

Hills, B. L. (1980). Vision, visibility, and perception in driving. Perception, 9(2), 183216. https://doi.org/10.1068/p090183

Hoffman, L., McDowd, J. M., Atchley, P., \& Dubinsky, R. (2005). The role of visual attention in predicting driving impairment in older adults. Psychology and Aging, 20(4), 610-622. https://doi.org/10.1037/0882-7974.20.4.610

Kau, S., Strumpf, H., Merkel, C., Stoppel, C. M., Heinze, H. J., Hopf, J. M., \& Schoenfeld, M. A. (2013). Distinct neural correlates of attending speed vs. 
coherence of motion. Neurolmage, 64(1), 299-327.

https://doi.org/10.1016/j.neuroimage.2012.08.080

Kaup, A. R., Drummond, S. P. A., \& Eyler, L. T. (2014). Brain Functional Correlates of Working Memory: Reduced Load-Modulated Activation and Deactivation in Aging without Hyperactivation or Functional Reorganization. Journal of the International Neuropsychological Society, 20(9), 945-950. https://doi.org/10.1017/S1355617714000824

Kennedy, K. M., Boylan, M. A., Rieck, J. R., Foster, C. M., \& Rodrigue, K. M. (2017). Dynamic range in BOLD modulation: lifespan aging trajectories and association with performance. Neurobiology of Aging, 60, 153-163. https://doi.org/10.1016/j.neurobiolaging.2017.08.027

Lappin, J. S., Bell, H. H., Harm, O. J., \& Kottas, B. (1975). On the relation between time and space in the visual discrimination of velocity. Journal of Experimental Psychology. Human Perception and Performance, 1(4), 383-394.

Lee, H. C., Cameron, D., \& Lee, A. H. (2003). Assessing the driving performance of older adult drivers: On-road versus simulated driving. Accident Analysis and Prevention, 35(5), 797-803. https://doi.org/10.1016/S0001-4575(02)00083-0

Lee, H. C., Drake, V., \& Cameron, D. (2002). Feature Article Identification of appropriate assessment criteria to measure older adults ' driving performance in simulated driving. Australian Occupational Therapy Journal, (November 2001), $138-145$.

Leung, H. C., Oh, H., Ferri, J., \& Yi, Y. (2007). Load response functions in the human spatial working memory circuit during location memory updating. Neurolmage, 35(1), 368-377. https://doi.org/10.1016/j.neuroimage.2006.12.012 
Eudave et al.

McKee, S. P. (1981). A local mechanism for differential velocity detection. Vision

Research, 21(4), 491-500. https://doi.org/10.1016/0042-6989(81)90095-X

Nagel, I. E., Preuschhof, C., Li, S.-C., Nyberg, L., Bäckman, L., Lindenberger, U., \& Heekeren, H. R. (2009). Performance level modulates adult age differences in brain activation during spatial working memory. Proceedings of the National Academy of Sciences of the United States of America, 106(52), 22552-7. https://doi.org/10.1073/pnas.0908238106

Norman, J. F., Ross, H. E., Hawkes, L. M., \& Long, J. R. (2003). Aging and the perception of speed. Perception, 32(1), 85-96. https://doi.org/10.1068/p3478

Oldfield, R. C. (1971). The assessment and analysis of handedness: The Edinburgh inventory. Neuropsychologia, 9(1), 97-113. https://doi.org/10.1016/0028$3932(71) 90067-4$

Orban, G. A., de Wolf, J., \& Maes, H. (1984). Factors influencing velocity coding in the human visual system. Vision Research, 24(1), 33-39. https://doi.org/10.1016/0042-6989(84)90141-X

Orban, G. A., Dupont, P., De Bruyn, B., Vandenberghe, R., Rosier, A., \& Mortelmans, L. (1998). Human brain activity related to speed discrimination tasks. Experimental Brain Research, 122(1), 9-22. https://doi.org/10.1007/s002210050486

Park, D. C., Polk, T. a, Hebrank, A. C., \& Jenkins, L. J. (2010). Age differences in default mode activity on easy and difficult spatial judgment tasks. Frontiers in Human Neuroscience, 3(January), 75. https://doi.org/10.3389/neuro.09.075.2009

Peirce, J. W. (2008). Generating stimuli for neuroscience using PsychoPy. Frontiers 
in Neuroinformatics, 2(January), 1-8. https://doi.org/10.3389/neuro.11.010.2008

Persson, J., Pudas, S., Nilsson, L.-G., \& Nyberg, L. (2014). Longitudinal assessment of default-mode brain function in aging. Neurobiology of Aging, 35(9), 21072117. https://doi.org/10.1016/j.neurobiolaging.2014.03.012

Prakash, R. S., Heo, S., Voss, M. W., Patterson, B., \& Kramer, A. F. (2012). Agerelated differences in cortical recruitment and suppression: Implications for cognitive performance. Behavioural Brain Research, 230(1), 192-200. https://doi.org/10.1016/j.bbr.2012.01.058

Raghuram, A., Lakshminarayanan, V., \& Khanna, R. (2005). Psychophysical estimation of speed discrimination. II. Aging effects. Journal of the Optical Society of America. A, Optics, Image Science, and Vision, 22(10), 2269-2280. https://doi.org/10.1364/JOSAA.22.002269

Raichle, M. E. (2015). The Brain's Default Mode Network. Annual Review of Neuroscience, 38(1), 433-447. https://doi.org/10.1146/annurev-neuro-071013014030

Raichle, M. E., MacLeod, A. M., Snyder, A. Z., Powers, W. J., Gusnard, D. A., \& Shulman, G. L. (2001). A default mode of brain function. Proceedings of the National Academy of Sciences of the United States of America, 98(2), 676-82. https://doi.org/10.1073/pnas.98.2.676

Reuter-Lorenz, P. A., \& Campbell, K. A. (2008). Neurocognitive ageing and the Compensation Hypothesis. Current Directions in Psychological Science, 17(3), 177-182.

Reuter-Lorenz, P. A., \& Park, D. C. (2014). How Does it STAC Up? Revisiting the Scaffolding Theory of Aging and Cognition. Neuropsychology Review. 
https://doi.org/10.1007/s11065-014-9270-9

Eudave et al.

Rieck, J. R., Rodrigue, K. M., Boylan, M. A., \& Kennedy, K. M. (2017). Age-related reduction of BOLD modulation to cognitive difficulty predicts poorer task accuracy and poorer fluid reasoning ability. Neurolmage, 147(July 2016), 262271. https://doi.org/10.1016/j.neuroimage.2016.12.022

Roenker, D. L., Cissell, G. M., Ball, K. K., Wadley, V. G., \& Edwards, J. D. (2003). Speed-of-processing and driving simulator training result in improved driving performance. Human Factors, 45(2), 218-233.

https://doi.org/10.1518/hfes.45.2.218.27241

Sambataro, F., Murty, V. P., Callicott, J. H., Tan, H. Y., Das, S., Weinberger, D. R., \& Mattay, V. S. (2010). Age-related alterations in default mode network: Impact on working memory performance. Neurobiology of Aging, 31(5), 839-852. https://doi.org/10.1016/j.neurobiolaging.2008.05.022

Scialfa, C. T., Guzy, L. T., Leibowitz, H. W., Garvey, P. M., \& Tyrrell, R. A. (1991). Age differences in estimating vehicle velocity. Psychology and Aging, 6(1), 6066. https://doi.org/10.1037/0882-7974.6.1.60

Shanmugaratnam, S., Kass, S. J., \& Arruda, J. E. (2010). Age differences in cognitive and psychomotor abilities and simulated driving. Accident Analysis and Prevention, 42(3), 802-808. https://doi.org/10.1016/j.aap.2009.10.002

Siman-Tov, T., Bosak, N., Sprecher, E., Paz, R., Eran, A., Aharon-Peretz, J., \& Kahn, I. (2017). Early age-related functional connectivity decline in high-order cognitive networks. Frontiers in Aging Neuroscience, 8(JAN), 1-17. https://doi.org/10.3389/fnagi.2016.00330

Snowden, R. J., \& Kavanagh, E. (2006). Motion perception in the ageing visual 
Eudave et al.

system: Minimum motion, motion coherence, and speed discrimination

thresholds. Perception, 35(1), 9-24. https://doi.org/10.1068/p5399

Stinchcombe, A., Gagnon, S., Zhang, J., Montembeault, P., \& Bedard, M. (2011).

Fluctuating Attentional Demand in a Simulated Driving Assessment: The Roles of Age and Driving Complexity. Traffic Injury Prevention, 12(6), 576-587. https://doi.org/10.1080/15389588.2011.607479

Sunaert, S., Van Hecke, P., Marchal, G., \& Orban, G. A. (2000). Attention to Speed of Motion, Speed Discrimination, and Task Difficulty: An fMRI Study. Neurolmage, 11(6), 612-623. https://doi.org/10.1006/nimg.2000.0587

Urwyler, P., Gruber, N., Müri, R. M., Jäger, M., Bieri, R., Nyffeler, T., ... Nef, T. (2015). Age-dependent visual exploration during simulated day- and night driving on a motorway: a cross-sectional study. BMC Geriatrics, 15(1), 18. https://doi.org/10.1186/s12877-015-0015-2 
Eudave et al.

\section{Figure Legends}

Figure 1. Speed discrimination fMRI paradigm. ITI = Inter-Trial Interval, $R T=$ Response Time.

Figure 2. Neuropsychological scores. Results were transformed to z-scores and classified by domains. Asterisc indicates significant differences between groups after Bonferroni correction. $Y S=$ Young subjects, OS = Older subjects .

Figure 3. High speed discrimination performance results. a) Accuracy (AC) and b) Response Time (RT) compared by groups; boxes indicate median and the $25^{\text {th }}$ to $75^{\text {th }}$ percentile and whiskers the minimum and maximum values. c) AC and d) RT compared by SPD between stimuli and group. Upper and lower limits represent the standard error. $Y S=$ Young subjects, OS = Older subjects .

Figure 4. Task > Control common and differential activation patterns between groups. Color-coded activation (hot) and deactivation (winter) parametrical maps. Clusters were FWE-corrected at $\mathrm{p}<0.05$. YS = Young subjects; $\mathrm{OS}=$ Older subjects.

Figure 5. Seed-to-Voxel functional connectivity revealed higher connectivity between DMN areas in young adults. Seeds (black font) and their targets (red font) reveal that a-j) young subjects (YS) mainly show increased connectivity between DMN areas, while k-m) older subjects (OS) have higher connectivity between frontal, parietal and basal regions. Connectivity clusters were FDR-corrected at $\mathrm{p}<0.05$. SMA $=$ Supplementary motor area, $\mathrm{MCC}=$ Middle cingulate cortex, $\mathrm{MT}=$ Middle temporal cortex, $\mathrm{PCC}=$ Posterior cingulate cortex, LOC $=$ Lateral occipital cortex. 


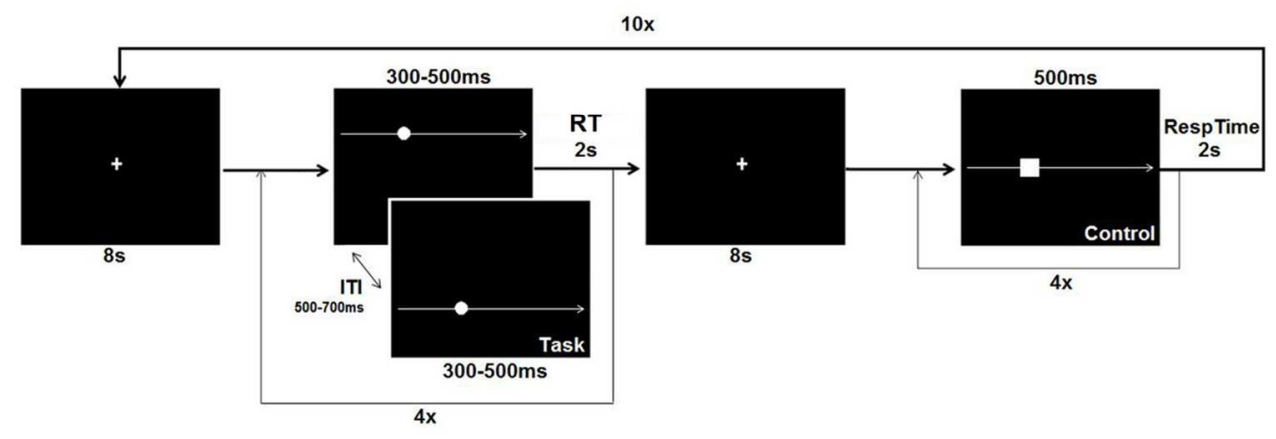

Figure 1. Speed discrimination fMRI paradigm. ITI = Inter-Trial Interval, RT $=$ Response Time.

$$
55 \times 18 \mathrm{~mm}(600 \times 600 \mathrm{DPI})
$$

John Wiley \& Sons, Inc. 


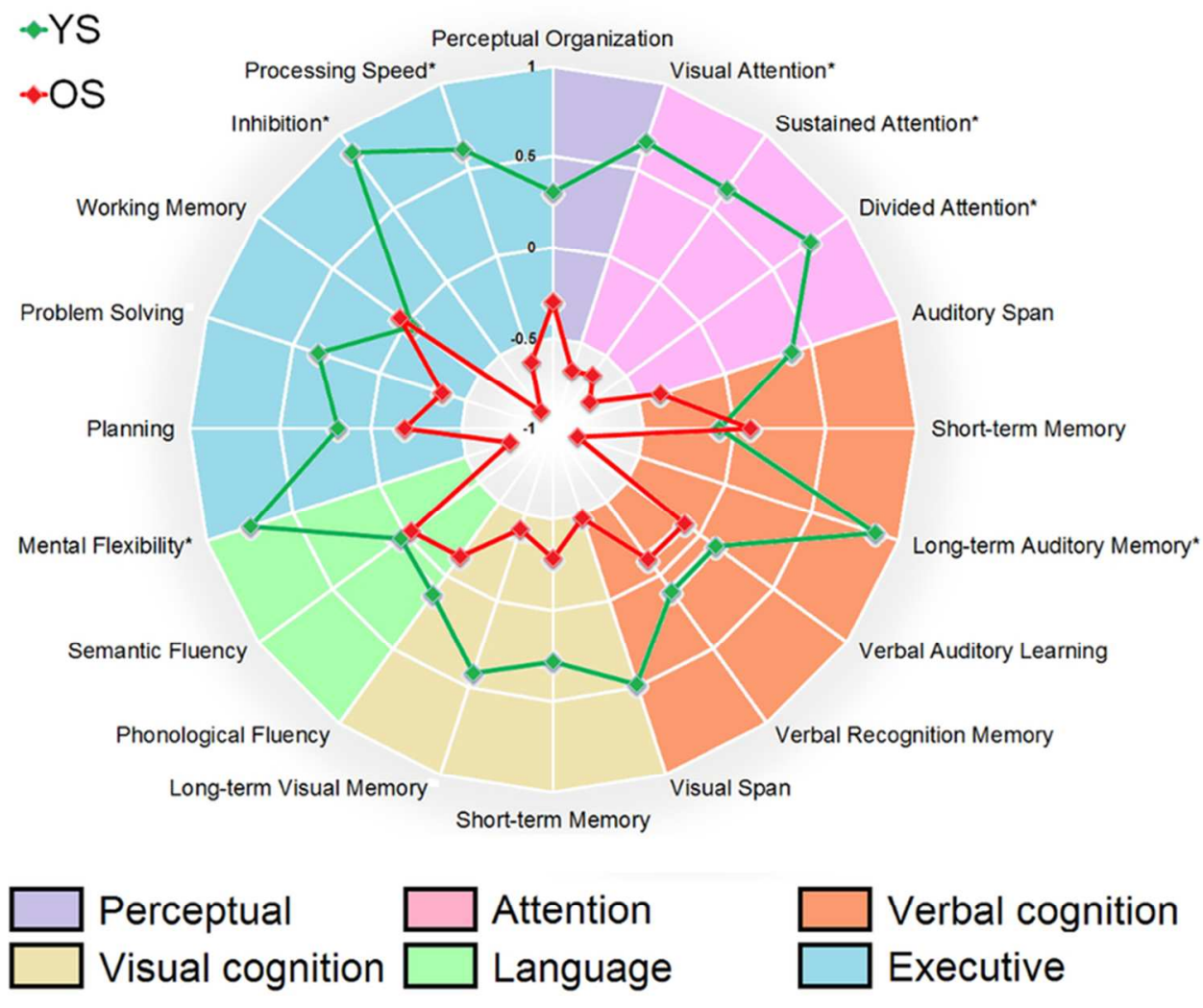

Figure 2. Neuropsychological scores. Results were transformed to z-scores and classified by domains. Asterisc indicates significant differences between groups after Bonferroni correction. YS = Young subjects, OS $=$ Older subjects.

$74 \times 62 \mathrm{~mm}(300 \times 300 \mathrm{DPI})$ 
AC
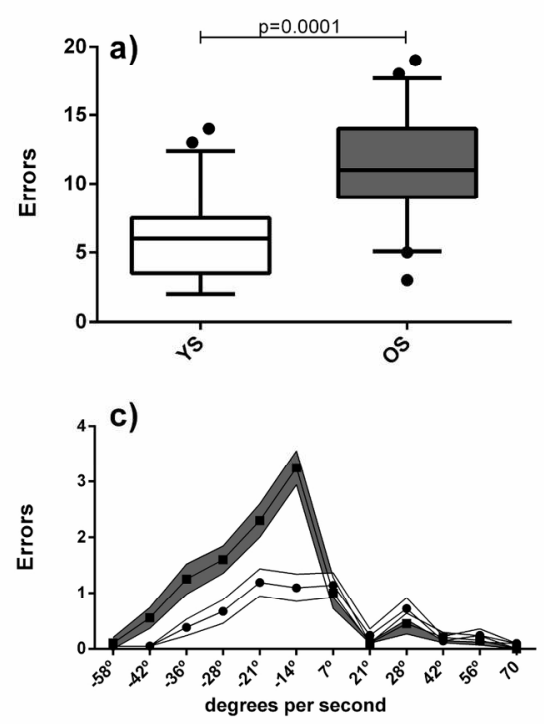

RT
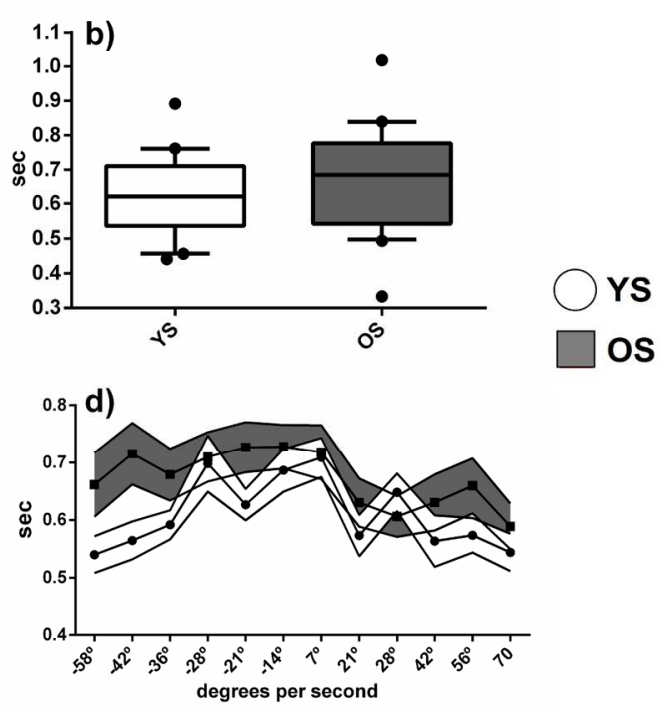

Figure 3. High speed discrimination performance results. a) Accuracy (AC) and b) Response Time (RT) compared by groups; boxes indicate median and the 25th to 75th percentile and whiskers the minimum and maximum values. c) AC and d) RT compared by SPD between stimuli and group. Upper and lower limits represent the standard error. YS = Young subjects, OS = Older subjects.

$101 \times 62 \mathrm{~mm}(600 \times 600$ DPI $)$ 

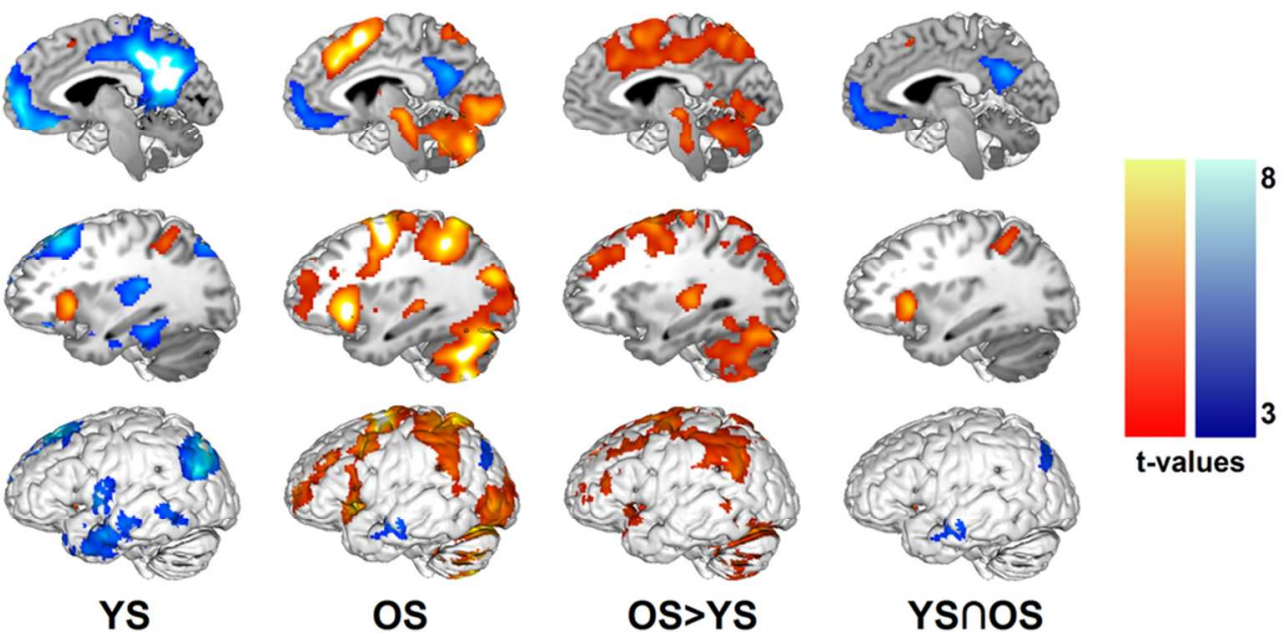

t-values

Figure 4. Task $>$ Control common and differential activation patterns between groups. Color-coded activation (hot) and deactivation (winter) parametrical maps. Clusters were FWE-corrected at $p<0.05$. YS = Young subjects; OS = Older subjects.

$81 \times 40 \mathrm{~mm}(300 \times 300$ DPI $)$ 

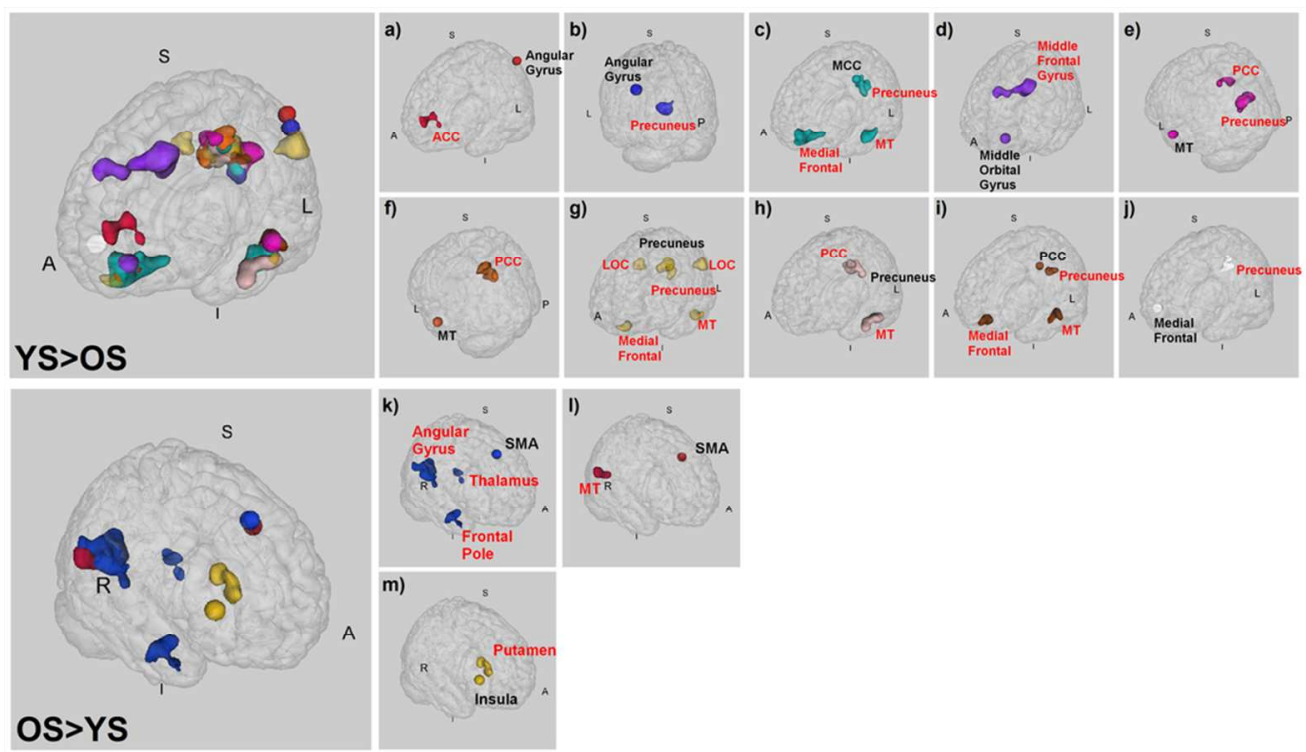

Figure 5. Seed-to-Voxel functional connectivity revealed higher connectivity between DMN areas in young adults. Seeds (black font) and their targets (red font) reveal that $a-j$ ) young subjects (YS) mainly show increased connectivity between DMN areas, while k-m) older subjects (OS) have higher connectivity between frontal, parietal and basal regions. Connectivity clusters were FDR-corrected at $p<0.05$. SMA $=$ Supplementary motor area, MCC = Middle cingulate cortex, MT = Middle temporal cortex, PCC $=$ Posterior cingulate cortex, LOC = Lateral occipital cortex.

$95 \times 54 \mathrm{~mm}(300 \times 300$ DPI $)$ 
Table I

Participant data and neuropsychological evaluation

\begin{tabular}{|c|c|c|c|c|c|}
\hline & YS & OS & S vs. OS & & \\
\hline & $M \pm \mathrm{SD}$ & $M \pm \mathrm{SD}$ & $W$-test & $p$-value & Effect size \\
\hline Gender (male/female) & $11 / 11$ & $14 / 6$ & 264 & 0.198 & 0.200 \\
\hline Age & $30.1 \pm 4.3$ & $67.4 \pm 5.2$ & 0.0 & $<.001^{*}$ & -1.000 \\
\hline Driving experience (years) & $8.9 \pm 4.6$ & $45.1 \pm 5.1$ & 0.0 & $<.001^{*}$ & -1.000 \\
\hline MMSE & $29.3 \pm 0.7$ & $28.9 \pm 1.2$ & 196.0 & 0.308 & 0.086 \\
\hline GDS & $2.1 \pm 1.9$ & $3.4 \pm 2.3$ & 126.0 & 0.059 & -0.302 \\
\hline Perceptual Organization & $50.5 \pm 8.8$ & $44.7 \pm 10.3$ & 245.0 & 0.059 & 0.360 \\
\hline Attention & $41.6 \pm 5.7$ & $55.4 \pm 9.2$ & 44.0 & $<.001^{*}$ & -0.780 \\
\hline Visual Attention & $20.6 \pm 5.9$ & $30 \pm 8.5$ & 72.0 & $<.001^{*}$ & -0.657 \\
\hline Sustained Attention & $60.6 \pm 8.6$ & $71.1 \pm 8.5$ & 96.0 & $0.002^{*}$ & -0.543 \\
\hline Divided Attention & $45.8 \pm 9.1$ & $65.9 \pm 20.7$ & 70.0 & $<.001^{*}$ & -0.667 \\
\hline Verbal Cognition & $10.6 \pm 0.7$ & $9.6 \pm 1.2$ & 277.0 & 0.005 & 0.532 \\
\hline Memory Span & $7.5 \pm 1.9$ & $6.1 \pm 1.8$ & 242.5 & 0.068 & 0.343 \\
\hline Short-term Memory & $10.4 \pm 2$ & $10.8 \pm 2.6$ & 190.0 & 0.607 & -0.095 \\
\hline Long-term Memory & $13.8 \pm 1.6$ & $10.1 \pm 2.8$ & 314.0 & $<.001^{*}$ & 0.740 \\
\hline Verbal Learning & $6.8 \pm 2$ & $6.3 \pm 2.5$ & 198.0 & 0.615 & 0.097 \\
\hline Verbal Recognition & $14.8 \pm 0.4$ & $14.5 \pm 0.8$ & 212.0 & 0.222 & 0.175 \\
\hline Visual Cognition & $9.4 \pm 1.5$ & $7.9 \pm 2.2$ & 257.5 & 0.025 & 0.427 \\
\hline Memory Span & $8.7 \pm 0.7$ & $8 \pm 0.7$ & 271.5 & 0.004 & 0.504 \\
\hline Short-term Memory & $10.4 \pm 1.9$ & $9 \pm 2.6$ & 235.0 & 0.108 & 0.302 \\
\hline Long-term Memory & $9.3 \pm 2.6$ & $6.6 \pm 3.9$ & 254.0 & 0.032 & 0.407 \\
\hline Language & $21.2 \pm 3.8$ & $21.8 \pm 4.4$ & 166.5 & 0.693 & -0.078 \\
\hline Phonological Fluency & $18.4 \pm 5.4$ & $19.8 \pm 5.3$ & 155.0 & 0.464 & -0.141 \\
\hline Semantic Fluency & $24.1 \pm 3.5$ & $23.8 \pm 4.4$ & 180.5 & 1.000 & 0.000 \\
\hline Executive Functions & $79.7 \pm 13.9$ & $112.2 \pm 26.9$ & 35.0 & $<.001^{*}$ & -0.806 \\
\hline Mental Flexibility & $43.9 \pm 8.6$ & $65.9 \pm 20.7$ & 63.0 & $<.001^{*}$ & -0.685 \\
\hline Planning & $103.5 \pm 63.8$ & $134.4 \pm 104.7$ & 122.0 & 0.493 & -0.144 \\
\hline
\end{tabular}




$\begin{array}{lccccc}\text { Problem Solving } & 65.7 \pm 52.1 & 119.1 \pm 84.4 & 90.0 & 0.008 & -0.501 \\ \text { Working Memory } & 8.6 \pm 2.3 & 9 \pm 2.7 & 200.5 & 0.813 & -0.045 \\ \text { Inhibition } & 99.2 \pm 12 & 123.4 \pm 16.6 & 50.5 & <.001^{*} & -0.760 \\ \text { Processing Speed } & 187.3 \pm 23.1 & 228.9 \pm 44.6 & 264.0 & 0.002^{*} & -0.587\end{array}$

Note. Mann-Whitney U test. Effect size by rank-biserial correlation.

*Significant values after Bonferroni correction.

MMSE=mini mental status examination, GDS=geriatric depression scale,

YS = Young Subjects, OS = Older Subjects

John Wiley \& Sons, Inc. 
Table II

Driving simulator factor and telemetry data

\begin{tabular}{|c|c|c|c|c|c|}
\hline & YS & OS & IS vs. OS & & \\
\hline & $M \pm \mathrm{SD}$ & $M \pm \mathrm{SD}$ & $W$-test & $p$-value & Effect size \\
\hline Speed & $-1850.9 \pm 248.6$ & $-2251.9 \pm 337$ & 285 & $<.001^{*}$ & 0.676 \\
\hline Total Session Time & $2043.3 \pm 236$ & $2426.5 \pm 330$ & 53 & $<.001^{*}$ & -0.688 \\
\hline$\%$ Time above speed limit & $4.4 \pm 3.6$ & $1.7 \pm 1.2$ & 269 & 0.003 & 0.582 \\
\hline Mean speed at $40 \mathrm{~km} / \mathrm{h}$ zone & $31.6 \pm 3.5$ & $26.8 \pm 6.2$ & 256 & 0.009 & 0.506 \\
\hline Mean speed at $50 \mathrm{~km} / \mathrm{h}$ zone & $16.5 \pm 3.1$ & $14.4 \pm 1.9$ & 245 & 0.023 & 0.441 \\
\hline Mean speed at $80 \mathrm{~km} / \mathrm{h}$ zone & $49.9 \pm 6.2$ & $43.3 \pm 5.8$ & 268 & 0.003 & 0.576 \\
\hline Mean speed at $120 \mathrm{~km} / \mathrm{h}$ zone & $94 \pm 12.8$ & $76.1 \pm 12$ & 285 & $<.001^{*}$ & 0.676 \\
\hline Yellow traffic light skips & $1.8 \pm 1.3$ & $3.2 \pm 2$ & 88 & 0.011 & -0.482 \\
\hline Dexterity & $92.1 \pm 25.8$ & $114.8 \pm 81.1$ & 150 & 0.552 & -0.118 \\
\hline $270-360^{\circ}$ steers in 0.5 seconds & $0.1 \pm 0.3$ & $0.6 \pm 1.3$ & 144 & 0.226 & -0.153 \\
\hline $180-270^{\circ}$ steers in 0.5 seconds & $2.7 \pm 2.6$ & $4.8 \pm 8.3$ & 143.5 & 0.420 & -0.156 \\
\hline $90-180^{\circ}$ steers in 0.5 seconds & $37.8 \pm 14.2$ & $46.9 \pm 37$ & 157 & 0.703 & -0.076 \\
\hline $60-90^{\circ}$ steers in 0.5 seconds & $58.1 \pm 17.6$ & $71 \pm 42.3$ & 134 & 0.279 & -0.212 \\
\hline Collision Risk & $50.9 \pm 53.8$ & $40.3 \pm 30.3$ & 173 & 0.939 & 0.018 \\
\hline Time w/gas pedal $>75 \%$ & $60.6 \pm 63.8$ & $48.4 \pm 36.5$ & 172.5 & 0.951 & 0.015 \\
\hline$\%$ Time w/gas pedal $>75 \%$ & $1.6 \pm 1.9$ & $1 \pm 0.8$ & 188 & 0.594 & 0.106 \\
\hline Collision w/other car & $0.5 \pm 0.9$ & $0.4 \pm 0.7$ & 173.5 & 0.910 & 0.021 \\
\hline Hard Braking & $-54.8 \pm 7.8$ & $-53.2 \pm 18.7$ & 198 & 0.402 & 0.165 \\
\hline$\%$ Time moving & $87.7 \pm 2.9$ & $88.0 \pm 5.6$ & 128 & 0.206 & -0.247 \\
\hline Time w/brake pedal $>75 \%$ & $3.5 \pm 7.5$ & $5.3 \pm 16.6$ & 162 & 0.800 & -0.047 \\
\hline$\%$ Time w/brake pedal $>75 \%$ & $0.1 \pm 0.2$ & $0.1 \pm 0.3$ & 172 & 0.959 & 0.012 \\
\hline Braking & $257 \pm 89.6$ & $261.2 \pm 153.3$ & 188 & 0.594 & 0.106 \\
\hline Time braking & $253 \pm 87.8$ & $260.4 \pm 154.6$ & 186 & 0.637 & 0.094 \\
\hline$\%$ Time braking & $12.6 \pm 4.8$ & $10.7 \pm 5.7$ & 221 & 0.124 & 0.300 \\
\hline
\end{tabular}


1

2

3

4

5

6

7

8

9

10

11

12

13

14

15

16

17

18

19

20

21

22

23

24

25

26

27

28

29

30

31

32

33

34

35

36

37

38

39

40

41

42

43

44

45

46

47

48

49

50

51

52

53

54

55

56

57

58

59

60 $\begin{array}{llllll}>10 \text { second brakes } & 6.4 \pm 3 & 4.6 \pm 3 & 231.5 & 0.062 & 0.362\end{array}$

$\begin{array}{cccccc}\text { Mean speed at } 100 \mathrm{~km} / \mathrm{h} \text { zone } & 35.4 \pm 21.8 & 37.6 \pm 24.3 & 160 & 0.772 & -0.059 \\ \text { Two wheel sidewalk invasion } & 57.3 \pm 24.7 & 71.6 \pm 17.8 & 82 & 0.008 & -0.518 \\ >5 \text { second brakes } & 2.3 \pm 1.9 & 1.8 \pm 1.3 & 188 & 0.584 & 0.106 \\ \text { Collision w/other objects } & 3.7 \pm 3.8 & 5 \pm 3.8 & 124.5 & 0.167 & -0.268 \\ \text { Red traffic light skips } & 1.9 \pm 2.1 & 3.8 \pm 6.5 & 123 & 0.140 & -0.276 \\ \text { Run overs } & 0.1 \pm 0.5 & 0.2 \pm 0.4 & 149 & 0.329 & -0.124\end{array}$

Note. Mann-Whitney U test. Effect size by rank-biserial correlation.

*Significant values after Bonferroni correction

YS = Young Subjects, OS = Older Subjects

John Wiley \& Sons, Inc. 


\section{Table III}

Hyperactivation clusters in OS

MNI Coordinates

\begin{tabular}{|c|c|c|c|c|c|c|}
\hline Region & BA & Cluster size & $x$ & $y$ & $z$ & T value \\
\hline Left Precentral Gyrus & 6 & 40497 & -22 & -22 & 66 & 7.23 \\
\hline Left Precentral Gyrus & 6 & & -38 & 4 & 38 & 6.27 \\
\hline Right SMA & 6 & & 4 & -4 & 54 & 6.19 \\
\hline Left Insula & 13 & & -32 & -24 & 4 & 6.09 \\
\hline Right Superior Frontal Gyrus & 6 & & 34 & -4 & 66 & 6.06 \\
\hline Left Middle Frontal Gyrus & 6 & & -28 & 2 & 58 & 6.04 \\
\hline Right SMA & 6 & & -12 & 2 & 70 & 5.97 \\
\hline Right Precuneus & 7 & & 6 & -58 & 52 & 5.96 \\
\hline Cerebellar Lobule I-IV (Vermis) & N/A & & 0 & -52 & -22 & 5.94 \\
\hline Left Fusiform Gyrus & 19 & & -34 & -66 & -18 & 5.94 \\
\hline Left Cerebellum Lobule VI & 19 & & -24 & -70 & -18 & 5.93 \\
\hline Left IFG p. Triangularis & 45 & 308 & -54 & 18 & -2 & 4.39 \\
\hline Left Temporal Pole & 22 & & -50 & 10 & -10 & 3.98 \\
\hline Left Temporal Pole & 22 & & -54 & 8 & -8 & 3.96 \\
\hline Left Temporal Pole & 47 & & -42 & 20 & -16 & 3.91 \\
\hline
\end{tabular}




\begin{tabular}{|c|c|c|c|c|c|c|c|c|c|c|c|}
\hline \multicolumn{12}{|c|}{ Conjunction analysis clusters of activation and connectivity } \\
\hline \multirow[b]{2}{*}{ Contrast/Region } & \multirow[b]{2}{*}{ BA } & \multicolumn{5}{|c|}{ MNI Coordinates } & \multicolumn{2}{|c|}{ Connected to } & \multicolumn{3}{|c|}{ MNI Coordinates } \\
\hline & & Cluster size & $\mathrm{x}$ & $\mathrm{y}$ & $\mathrm{z}$ & T value & $\mathrm{YS}>\mathrm{OS}$ & OS>YS & $\mathrm{x}$ & $\mathrm{y}$ & $\mathrm{z}$ \\
\hline \multicolumn{12}{|l|}{ YSกOS (pos) } \\
\hline Right Precentral Gyrus & 6 & 729 & 46 & 6 & 28 & 7.36 & \multirow[t]{5}{*}{ Right Superior Frontal Gyrus } & & 16 & 4 & 66 \\
\hline Right Insula Lobe & 13 & 500 & 32 & 22 & 2 & 6.46 & & Left Putamen & 32 & 22 & 2 \\
\hline \multirow[t]{3}{*}{ Right Supplementary Motor Area } & 8 & 358 & 4 & 16 & 50 & 6.46 & & Right Angular Gyrus & 42 & -50 & 18 \\
\hline & & & & & & & & Right Temporal Pole & 44 & 0 & -28 \\
\hline & & & & & & & & Left Thalamus & -16 & -32 & 0 \\
\hline Left Supplementary Motor Area & 6 & & -8 & 14 & 46 & 4.2 & & Right Midde Temporal Gyrus & 54 & -56 & 6 \\
\hline
\end{tabular}

\section{YSnOS (neg)}

$\begin{array}{rccccccl}\text { Left Anterior Cingulate } & 32 & 1473 & -8 & 52 & 0 & 5.69 & \\ \text { Left Mid Orbital Gyrus } & 10 & & -10 & 44 & -10 & 5.31 & \text { Left Middle Frontal Gyrus } \\ & & & 4 & 56 & 6 & 4.95 & \text { Precuneus } \\ \text { Left Precuneus } & 23 & 1455 & -4 & -56 & 26 & 6.67 & \text { Precuneus } \\ & & & & & & & \text { Right Lateral Occipital Cortex } \\ & & & & & & \text { Left Lateral Occipital Cortex }\end{array}$

$\begin{array}{ccc}-26 & 36 & 46 \\ 6 & -56 & 22 \\ 6 & -44 & 26 \\ 52 & -66 & 34 \\ -46 & -66 & 28\end{array}$




\begin{tabular}{|c|c|c|c|c|c|c|c|c|c|c|}
\hline & & & & & & & Frontal Medial Cortex & -4 & 54 & -16 \\
\hline & & & & & & & Left Middle Temporal Gyrus & -54 & -10 & -22 \\
\hline \multirow[t]{3}{*}{ Left Middle Cingulate } & 23 & & -8 & -44 & 34 & 5.9 & Frontal Medial Cortex & 2 & 46 & -16 \\
\hline & & & & & & & Precuneus & -2 & -60 & 8 \\
\hline & & & & & & & Left Middle Temporal Gyrus & -54 & -8 & -24 \\
\hline \multirow[t]{2}{*}{ Left Precuneus } & 23 & & -8 & -56 & 16 & 5.88 & Posterior Cingulate & 6 & -44 & 26 \\
\hline & & & & & & & Left Middle Temporal Gyrus & -60 & -10 & -24 \\
\hline \multirow[t]{3}{*}{ Right Posterior Cingulate } & 23 & & 6 & -50 & 28 & 5.27 & Frontal Medial Cortex & 2 & 44 & -16 \\
\hline & & & & & & & Precuneus & -6 & -54 & 20 \\
\hline & & & & & & & Left Middle Temporal Gyrus & -56 & -18 & -14 \\
\hline Left Angular Gyrus & 39 & 434 & -38 & -76 & 46 & 5.04 & Left Paracingulate Gyrus & -12 & 40 & 4 \\
\hline Left Angular Gyrus & 39 & & -42 & -72 & 38 & 4.84 & Precuneus & -4 & -60 & 8 \\
\hline eft Middle Temporal Gyrus & 21 & 309 & -60 & -14 & -8 & 5.07 & & & & \\
\hline \multirow[t]{2}{*}{ eft Middle Temporal Gyrus } & 21 & & -62 & -10 & -12 & 5.06 & Precuneus & -10 & -68 & 20 \\
\hline & & & & & & & Posterior Cingulate & -8 & -36 & 40 \\
\hline eft Middle Temporal Gyrus & 21 & & -56 & -14 & -18 & 4.54 & Posterior Cingulate & 12 & -44 & 28 \\
\hline eft Middle Temporal Gyrus & 21 & & -58 & -4 & -18 & 4.31 & Left Frontal Pole & -32 & 48 & 8 \\
\hline
\end{tabular}

BA $=$ Broadmann Area, YS $=$ Young Subjects, OS $=$ Older Subjects 


\begin{tabular}{|c|c|c|c|c|c|c|}
\hline \multicolumn{7}{|c|}{ BOLD activation behavioral correlates } \\
\hline & YS & $r$ & $p$-value & OS & $r$ & $p$-value \\
\hline \multicolumn{7}{|l|}{ Performance } \\
\hline \multirow[t]{7}{*}{ AC } & Right Precentral Gyrus & -0.449 & 0.036 & Right Superior Medial Gyrus* & -0.534 & 0.015 \\
\hline & Right IFG p. Opercularis & -0.481 & 0.024 & Left Precuneus* & -0.551 & 0.012 \\
\hline & Right Inferior Parietal Lobule & -0.575 & 0.005 & Left Middle Cingulate* $^{*}$ & -0.499 & 0.025 \\
\hline & Left Inferior Parietal Lobule & -0.428 & 0.047 & Right Posterior Cingulate* & -0.535 & 0.015 \\
\hline & Right Insula Lobe & -0.509 & 0.015 & - & & \\
\hline & Left Insula Lobe & -0.532 & 0.011 & - & & \\
\hline & Right Supplementary Motor Area & -0.481 & 0.023 & & & \\
\hline \multicolumn{7}{|c|}{ Neuropsychological } \\
\hline Attention & Right Supramarginal Gyrus & -0.444 & 0.050 & - & & \\
\hline \multirow[t]{2}{*}{ Verbal } & Left Precuneus* & -0.487 & 0.034 & - & & \\
\hline & Right Precuneus* & -0.510 & 0.026 & - & & \\
\hline Language & Right Superior Medial Gyrus* & 0.672 & 0.002 & & & \\
\hline Executive & Right IFG p. Opercularis & -0.469 & 0.043 & Left Inferior Parietal Lobule & 0.512 & 0.025 \\
\hline \multirow[t]{2}{*}{ Perception } & Left Mid Orbital Gyrus* & -0.512 & 0.025 & - & & \\
\hline & Left Middle Temporal Gyrus* & -0.459 & 0.048 & - & & \\
\hline
\end{tabular}




\section{Driving Simulator}

\begin{tabular}{|c|c|c|c|c|c|c|}
\hline \multirow[t]{2}{*}{ Speed } & Left Angular Gyrus* & -0.485 & 0.030 & - & & \\
\hline & Left Angular Gyrus* & -0.471 & 0.036 & - & & \\
\hline Dexterity & Left Mid Orbital Gyrus* & 0.492 & 0.027 & - & & \\
\hline Collision Risk & Left Insula Lobe & 0.491 & 0.028 & Right Superior Medial Gyrus* & 0.522 & 0.032 \\
\hline \multirow[t]{4}{*}{ Braking } & Right IFG p. Opercularis & 0.483 & 0.031 & Left Inferior Parietal Lobule & 0.491 & 0.045 \\
\hline & Right Supramarginal Gyrus & 0.635 & 0.003 & - & & \\
\hline & Right Supplementary Motor Area & 0.519 & 0.019 & - & & \\
\hline & Left Supplementary Motor Area & 0.497 & 0.026 & - & & \\
\hline \multirow[t]{4}{*}{ Hard Braking } & Left Superior Parietal Lobule & 0.499 & 0.025 & - & & \\
\hline & Left Mid Orbital Gyrus* & -0.637 & 0.003 & & & \\
\hline & Left Middle Temporal Gyrus* & -0.612 & 0.004 & & & \\
\hline & Left Middle Temporal Gyrus* & -0.468 & 0.037 & & & \\
\hline
\end{tabular}

Note. In the AC, Attention and Executive variables, negative $r$ values are associated to to better performance with increased activation.

${ }^{*}$ Regions from the negative conjunction analysis 


\section{Table VI}

Functional connectivity behavioral correlates

YS

OS

\begin{tabular}{|c|c|c|c|c|c|c|c|c|}
\hline & Seed & Target & $r$ & $p$-value & Seed & Target & $r$ & $p$-value \\
\hline \multicolumn{9}{|l|}{ Performance } \\
\hline \multirow[t]{2}{*}{$A C$} & Right Precentral Gyrus & Right Superior Frontal Gyrus & 0.478 & 0.024 & Right Supplementary Motor Area & Left Thalamus & -0.471 & 0.036 \\
\hline & Left Middle Temporal Gyrus* & Posterior Cingulate & -0.585 & 0.004 & & & & \\
\hline \multicolumn{9}{|c|}{ Neuropsychological } \\
\hline \multirow[t]{2}{*}{ Attention } & Left Precuneus* & Precuneus & -0.533 & 0.016 & - & - & - & - \\
\hline & Left Middle Temporal Gyrus* & Precuneus & -0.614 & 0.004 & - & - & - & - \\
\hline \multirow[t]{2}{*}{ Language } & Left Middle Temporal Gyrus* & Precuneus & 0.506 & 0.027 & Right Supplementary Motor Area & Right Angular Gyrus & 0.563 & 0.012 \\
\hline & Right Posterior Cingulate* & Frontal Medial Cortex & 0.475 & 0.040 & Left Supplementary Motor Area & Right Midde Temporal Gyrus & 0.479 & 0.038 \\
\hline \multicolumn{9}{|l|}{ Driving Simulator } \\
\hline Speed & - & - & - & - & Right Supplementary Motor Area & Right Angular Gyrus & -0.675 & 0.003 \\
\hline Dexterity & Right Superior Medial Gyrus* & Precuneus & 0.534 & 0.015 & - & - & - & - \\
\hline Acceleration & - & - & - & - & Right Supplementary Motor Area & Left Thalamus & 0.577 & 0.015 \\
\hline
\end{tabular}

Note. In the AC and Attention variables, negative $r$ values are associated to better performance with increased connectivity.

*Seeds from the negative conjunction analysis 